\title{
Thiosemicarbazones of Formyl Benzoic Acids as Novel Potent Inhibitors of Estrone Sulfatase
}

Peter Jütten, Winfried Schumann, Albert Härtl, Hans-Martin Dahse, ${ }^{*}$ and Udo Gräfe

Leibniz Institute for Natural Products Research and Infection Biology, HKI, Beutenbergstrasse 11a, 07745 Jena, Germany

\section{Contents}

Experimental section for compounds $\mathbf{2 - 4 0}$ and $\mathbf{4 5 - 4 9}$ including

${ }^{1} \mathrm{H}$ and ${ }^{13} \mathrm{C}$ NMR spectroscopic data of the compounds

$\mathrm{S} 2$

Elemental analysis data of selected compounds

S12

References

S13

* Corresponding author. Tel.: +49-3641-65-6878. Fax: +49-3641-65-6879. E-mail: hansmartin.dahse@hki-jena.de. 


\section{Experimental Section}

Chemical Compounds and Synthetic Procedures. General Information. The following substituted benzoic acids are available commercially: phthalaldehydic acid, isophthalaldehydic acid, 2-acetylbenzoic acid, 2-formyl-benzenesulfonic acid, 2-formylboronic acid, 3-formyl salicylic acid, and 5-formyl salicylic acid, as well as the 2-, 3-, and 4-hydroxybenzaldehydes. Minor modifications of literature procedures furnished: 3-hydroxy-7-methoxy-3H-isobenzofuran-1-one (41) ${ }^{1}$, 3-hydroxy-7-methoxy-6-methyl-3Hisobenzofuran-1-one (42) ${ }^{2}$, 3-hydroxy-7-methoxy-4-methyl-3H-isobenzofuran-1-one (43) ${ }^{2}$, 3-formyl-4hydroxy-benzoic acid $^{3}$, and 3-formyl-4-hydroxy-benzenesulfonic acid ${ }^{4}$. The thiosemicarbazides and semicarbazides are commercially available or were prepared by literature procedures. ${ }^{5}$

Analytical thin layer chromatography (TLC) was performed on precoated plates (Merck TLC aluminium sheets silica gel $60 \mathrm{~F}_{254}$ ). Products and starting material were detected by viewing under UV light (254 or $366 \mathrm{~nm})$. Column chromatography was performed on silica gel 60 (0.063-0.1 mm; Merck). Nuclear

magnetic resonance spectra (NMR) were obtained on a Bruker DPX $300\left({ }^{1} \mathrm{H}, 300 \mathrm{MHz} ;{ }^{13} \mathrm{C} 75 \mathrm{MHz}\right)$ and Bruker DRX $500\left({ }^{1} \mathrm{H}, 500 \mathrm{MHz} ;{ }^{13} \mathrm{C}, 125 \mathrm{MHz}\right)$ instruments; chemical shifts $(\delta)$ are expressed in parts per million ( $\mathrm{ppm}$ ) relative to the residual solvent peak. Electrospray mass spectra (ESMS) were obtained with a VG Quattro mass spectrometer. Melting points were taken on a Büchi B-540 melting point apparatus and are uncorrected. $\mathrm{pK}_{\mathrm{a}}$ measurements are obtained from the titration curve applying the Kolthoff method. 5-10 mg of the test compound was dissolved in 20\% aqueous DMF. This was then titrated against $10 \mathrm{mM} \mathrm{NaOH}$ solution via a 5-mL burette. Elemental analyses indicated by the symbols of the elements were within $\pm 0.4 \%$ of the theoretical values. Solvents were dried according to the standard procedures. Chemical yields were not optimized.

5-(3-Formylphenyl)-1H-tetrazole (40). To a mixture of 3-cyanobenzaldehyde (5.00 g, $38.1 \mathrm{mmol})$, triethylorthoformate $(6.15 \mathrm{~g}, 41.4 \mathrm{mmol})$, and $\mathrm{EtOH}(5.27 \mathrm{~g}, 114.3 \mathrm{mmol})$ was added a catalytic amount of conc. $\mathrm{HCl}(15 \mu \mathrm{l})$. The reaction is highly exothermic. The reaction mixture is boiled for a minute and then rapidly cooled down to room temperature. The mixture is made slightly alkaline by a few drops of alcoholic $\mathrm{KOH}$ solution, concentrated and distilled under vacuum to give 3-cyanobenzaldehyde diethyl acetal as a colorless liquid (7.30 g, 93\%); bp $160{ }^{\circ} \mathrm{C} / 0.015$ mbar. A solution of this nitrile (1.52 g, 7.5 mmol $)$ and tributyltin azide $(2.49 \mathrm{~g}, 7.5 \mathrm{mmol})$ in dry toluene $(6 \mathrm{~mL})$ were stirred under reflux overnight. 
The reaction mixture was cooled to room temperature and diluted with toluene $(6 \mathrm{~mL})$. Then $10 \mathrm{~N}$ aqueous $\mathrm{NaOH}$ (0.9 mL, 1.2 equiv) and triphenylmethyl chloride ( $2.19 \mathrm{~g}, 1.05$ equiv) were added, and the mixture was stirred at room temperature for $6 \mathrm{~h}$. The mixture was diluted with water $(12 \mathrm{~mL})$ and extracted with $\mathrm{CH}_{2} \mathrm{Cl}_{2}$. The combined organic phases were washed with water and brine, dried $\left(\mathrm{Na}_{2} \mathrm{SO}_{4}\right)$, filtered, and concentrated. The resulting syrup crystallized upon trituration with ethanol to give 5-(3diethoxymethylphenyl)-1-triphenylmethyl- $1 \mathrm{H}$-tetrazole $39(3.60 \mathrm{~g}, 98 \%)$ as a solid: $\mathrm{mp} 105-108{ }^{\circ} \mathrm{C} ;{ }^{1} \mathrm{H}-$ $\operatorname{NMR}\left(\mathrm{CDCl}_{3}\right) \delta 1.27(\mathrm{t}, J=7.0 \mathrm{~Hz}, 6 \mathrm{H}), 3.53-3.74(\mathrm{~m}, 4 \mathrm{H}), 5.57(\mathrm{~s}, 1 \mathrm{H}), 7.14-7.43(\mathrm{~m}, 15 \mathrm{H}), 7.48(\mathrm{t}, J$ $=7.7 \mathrm{~Hz}, 1 \mathrm{H}), 7.61,8.12(2 \mathrm{~d}, J=7.7 \mathrm{~Hz}, 2 \mathrm{H}), 8.27(\mathrm{~s}, 1 \mathrm{H})$. Anal. $\left(\mathrm{C}_{31} \mathrm{H}_{30} \mathrm{~N}_{4} \mathrm{O}_{2}\right) \mathrm{C}, \mathrm{H}, \mathrm{N}$.

A solution of 39 (1.80 g, $3.7 \mathrm{mmol}), 10 \%$ hydrochloric acid $(25 \mathrm{~mL})$, and tetrahydrofuran $(50 \mathrm{~mL})$ was stirred at room temperature for $4 \mathrm{~h}$. To the solution was added excess $10 \%$ aqueous sodium hydroxide and the solvents were removed under vacuum. The resulting residue was dissolved in water, and the mixture was filtered to remove the triphenylmethanol. The filtrate was acidified with dilute $\mathrm{HCl}$, and the white precipitate of 40 was recovered by filtration and dried $(0.45 \mathrm{~g}, 70 \%)$; mp 196-198 ${ }^{\circ} \mathrm{C} ;{ }^{1} \mathrm{H}-\mathrm{NMR}$ (DMSO$\left.d_{6}\right) \delta 7.83(\mathrm{t}, J=7.7 \mathrm{~Hz}, 1 \mathrm{H}), 8.10,8.34(2 \mathrm{~d}, J=7.7 \mathrm{~Hz}, 2 \mathrm{H}), 8.54(\mathrm{~s}, 1 \mathrm{H}), 10.11(\mathrm{~s}, 1 \mathrm{H}) ;{ }^{13} \mathrm{C}-\mathrm{NMR}$ $\left(\mathrm{DMSO}-d_{6}\right) \delta 125.37,127.30,130.39,132.08,132.40,136.92,192.62$. Anal. $\left(\mathrm{C}_{8} \mathrm{H}_{6} \mathrm{~N}_{4} \mathrm{O}\right) \mathrm{C}, \mathrm{H}, \mathrm{N}$.

\section{3,7-Dihydroxy-3H-isobenzofuran-1-one (45), 3,7-Dihydroxy-6-methyl-3H-isobenzofuran-1-one} (46), 3,7-Dihydroxy-4-methyl-3H-isobenzofuran-1-one (47), 3,7-Dihydroxy-6-methoxy-3Hisobenzofuran-1-one (48). The methyl ether (41-44; $5 \mathrm{mmol})$ was dissolved in dry $\mathrm{CH}_{2} \mathrm{Cl}_{2}(25 \mathrm{~mL})$ and cooled to $-78{ }^{\circ} \mathrm{C}$ under argon atmosphere. To the solution was added $1 \mathrm{M} \mathrm{BBr}_{3}(25 \mathrm{~mL})$ in $\mathrm{CH}_{2} \mathrm{Cl}_{2}$ over $20 \mathrm{~min}$, followed by stirring the mixture for $2 \mathrm{~h}$ at $-78^{\circ} \mathrm{C}$. The reaction mixture was warmed to $-10{ }^{\circ} \mathrm{C}$ and quenched by addition of water $(50 \mathrm{~mL})$. The aqueous phase was extracted with EtOAc. The combined organic phases were dried $\left(\mathrm{Na}_{2} \mathrm{SO}_{4}\right)$, filtered, and concentrated. The residue was chromatographed on silica gel with $\mathrm{CHCl}_{3}$ /acetone 20:1 to yield the products as white solids.

3,7-Dihydroxy-3H-isobenzofuran-1-one (45, 85\%). Anal. $\left(\mathrm{C}_{8} \mathrm{H}_{6} \mathrm{O}_{4}\right) \mathrm{C}, \mathrm{H}$.

3,7-Dihydroxy-6-methyl-3H-isobenzofuran-1-one (46, 90\%); mp 100-102 ${ }^{\circ} \mathrm{C}$. Anal. $\left(\mathrm{C}_{9} \mathrm{H}_{8} \mathrm{O}_{4}\right) \mathrm{C}$, H.

3,7-Dihydroxy-4-methyl-3H-isobenzofuran-1-one (47, 33\%). Anal. $\left(\mathrm{C}_{9} \mathrm{H}_{8} \mathrm{O}_{4}\right) \mathrm{C}, \mathrm{H}$.

3,7-Dihydroxy-6-methoxy-3H-isobenzofuran-1-one (48, 82\%); mp 149-151 ${ }^{\circ} \mathrm{C}$. Anal. $\left(\mathrm{C}_{9} \mathrm{H}_{8} \mathrm{O}_{5}\right) \mathrm{C}$, H. 
3,6,7-Trihydroxy-3H-isobenzofuran-1-one (49). To a solution of 48 (2 mmol) in dry $\mathrm{CH}_{2} \mathrm{Cl}_{2}(30$ $\mathrm{mL})$ at $0{ }^{\circ} \mathrm{C}$ was added $\mathrm{AlCl}_{3}(50 \mathrm{mmol})$. The mixture was warmed to room temperature, stirred for $2 \mathrm{~h}$, and quenched by the addition of ice water $(150 \mathrm{~mL})$. The aqueous layer was extracted with EtOAc. The combined organic phases were dried $\left(\mathrm{Na}_{2} \mathrm{SO}_{4}\right)$, filtered, and concentrated to give $49(299 \mathrm{mg}, 82 \%)$ as a white solid; mp $176-178^{\circ} \mathrm{C}$. Anal. $\left(\mathrm{C}_{8} \mathrm{H}_{6} \mathrm{O}_{5}\right) \mathrm{C}, \mathrm{H}$.

2-(4-Cyclohexylthiosemicarbazono)methyl-benzoic Acid (2). mp 232-234 ${ }^{\circ} \mathrm{C}$; ${ }^{1} \mathrm{H}-\mathrm{NMR}$ $\left(\mathrm{DMSO}-d_{6}\right) \delta 1.13-1.93(\mathrm{~m}, 10 \mathrm{H}), 4.17(\mathrm{~m}, 1 \mathrm{H}), 7.44-7.51(\mathrm{~m}, 1 \mathrm{H}), 7.54-7.62(\mathrm{~m}, 1 \mathrm{H}), 7.78-7.84(\mathrm{~m}$, $1 \mathrm{H}), 8.13(\mathrm{~d}, J=7.4 \mathrm{~Hz}, 1 \mathrm{H}), 7.94(\mathrm{~d}, J=8.5 \mathrm{~Hz}, 1 \mathrm{H}), 8.74(\mathrm{~s}, 1 \mathrm{H}), 11.53(\mathrm{~s}, 1 \mathrm{H}) ;{ }^{13} \mathrm{C}-\mathrm{NMR}$ (DMSO$\left.d_{6}\right) \delta 24.92,25.15,31.83,52.67,127.29,129.41,130.00,130.93,131.70,134.11,141.47,168.30,175.96$. Anal. $\left(\mathrm{C}_{15} \mathrm{H}_{19} \mathrm{~N}_{3} \mathrm{O}_{2} \mathrm{~S}\right) \mathrm{C}, \mathrm{H}, \mathrm{N}, \mathrm{S}$.

3-(4-Cyclohexylthiosemicarbazono)methyl-benzoic Acid (3). mp 239-241 ${ }^{\circ} \mathrm{C}$; ${ }^{1} \mathrm{H}-\mathrm{NMR}$ $\left(\mathrm{DMSO}_{6}\right) \delta 1.05-1.52(\mathrm{~m}, 5 \mathrm{H}), 1.54-1.79(\mathrm{~m}, 3 \mathrm{H}), 1.80-1.94(\mathrm{~m}, 2 \mathrm{H}), 4.07-4.27(\mathrm{~m}, 1 \mathrm{H}), 7.55(\mathrm{t}, J=$ $8.0 \mathrm{~Hz}, 1 \mathrm{H}, \mathrm{H}-5), 7.94$ (m, $1 \mathrm{H}, \mathrm{H}-6), 8.03-8.19$ (m, 3 H, CH=N, H-2,4), 11.46 (s, $1 \mathrm{H},=\mathrm{N}-\mathrm{NH}), 13.14$ (br, $1 \mathrm{H}) ;{ }^{13} \mathrm{C}-\mathrm{NMR}\left(\mathrm{DMSO}-d_{6}\right) \delta 24.97,25.15,31.76,52.78,128.47,130.80(\mathrm{C}-2,6), 129.03(\mathrm{C}-5)$, $130.43(\mathrm{C}-4), 131.38,134.57(\mathrm{C}-1,3), 140.46(\mathrm{CH}=\mathrm{N}), 169.96(\mathrm{COOH}), 175.78(\mathrm{C}=\mathrm{S})$. Anal. $\left(\mathrm{C}_{15} \mathrm{H}_{19} \mathrm{~N}_{3} \mathrm{O}_{2} \mathrm{~S}\right) \mathrm{C}, \mathrm{H}, \mathrm{N}, \mathrm{S}$.

4-(4-Cyclohexylthiosemicarbazono)methyl-benzoic Acid (4). mp 255-260 ${ }^{\circ} \mathrm{C}$; ${ }^{1} \mathrm{H}-\mathrm{NMR}$ $\left(\mathrm{DMSO}_{6}\right) \delta 1.11-1.35(\mathrm{~m}, 3 \mathrm{H}), 1.37-1.53(\mathrm{~m}, 2 \mathrm{H}), 1.55-1.65(\mathrm{~m}, 1 \mathrm{H}), 1.67-1.78(\mathrm{~m}, 2 \mathrm{H}), 1.81-1.92$ (m, $2 \mathrm{H}), 4.12-4.26(\mathrm{~m}, 1 \mathrm{H}), 7.87,7.94(2 \mathrm{~d}, J=8.5 \mathrm{~Hz}, 2 \mathrm{H}), 8.09$ (m, J = 8.5 Hz, $1 \mathrm{H}), 8.09$ (s, $1 \mathrm{H})$, $11.50(\mathrm{~s}, 1 \mathrm{H}) ;{ }^{13} \mathrm{C}-\mathrm{NMR}\left(\mathrm{DMSO}-d_{6}\right): \delta 24.93,25.11,31.71,52.82,127.25,128.57,131.37,138.20$, 140.90, 166.90, 175.88. Anal. $\left(\mathrm{C}_{15} \mathrm{H}_{19} \mathrm{~N}_{3} \mathrm{O}_{2} \mathrm{~S}\right) \mathrm{C}, \mathrm{H}, \mathrm{N}, \mathrm{S}$.

2-(4-Cyclohexylthiosemicarbazono)methyl-phenol (5). mp 195-196 ${ }^{\circ} \mathrm{C} ;{ }^{1} \mathrm{H}-\mathrm{NMR}$ (DMSO- $d_{6}$ ) $\delta$ 1.02-1.49 (m, 5 H), 1.53-1.64 (m, 1 H), 1.65-1.77 (m, 2 H), 1.81-1.93 (m, 2 H), 4.09-4.25 (m, 1 H), 6.79 (m, 1 H), $6.89(\mathrm{~m}, 1 \mathrm{H}), 7.21(\mathrm{~m}, 1 \mathrm{H}), 7.86(\mathrm{~m}, 1 \mathrm{H}), 7.91(\mathrm{~s}, 1 \mathrm{H}), 8.37$ (s, $1 \mathrm{H}), 9.90$ (s, $1 \mathrm{H}), 11.30$ (s, 1 $\mathrm{H}) ;{ }^{13} \mathrm{C}-\mathrm{NMR}\left(\mathrm{DMSO}-d_{6}\right) \delta 24.84,25.10,31.80,52.47,116.01,119.19,126.66,130.99,120.32,149.43$, 156.37, 175.61. Anal. $\left(\mathrm{C}_{14} \mathrm{H}_{19} \mathrm{~N}_{3} \mathrm{OS}\right) \mathrm{C}, \mathrm{H}, \mathrm{N}, \mathrm{S}$.

3-(4-Cyclohexylthiosemicarbazono)methyl-phenol (6). mp 186-188 ${ }^{\circ} \mathrm{C} ;{ }^{1} \mathrm{H}-\mathrm{NMR}$ (DMSO- $d_{6}$ ) $\delta$ 1.01-1.50 (m, 5 H), 1.52-1.64 (m, $1 \mathrm{H}), 1.64-1.77$ (m, $2 \mathrm{H}), 1.80-1.95$ (m, $2 \mathrm{H}), 4.07-4.26$ (m, $1 \mathrm{H}), 6.80$ (m, $1 \mathrm{H}), 7.10-7.24(\mathrm{~m}, 2 \mathrm{H}), 7.90(\mathrm{~d}, J=8.6 \mathrm{~Hz}, 1 \mathrm{H}), 7.96(\mathrm{~s}, 1 \mathrm{H}), 9.55$ (br, $1 \mathrm{H}), 11.30$ (br s, $1 \mathrm{H})$; 
${ }^{13} \mathrm{C}-\mathrm{NMR}\left(\mathrm{DMSO}-d_{6}\right) \delta 24.84,25.11,31.80,52.51,113.33,117.13,118.59,129.67,135.30,142.47$, 157.56, 175.72. Anal. $\left(\mathrm{C}_{14} \mathrm{H}_{19} \mathrm{~N}_{3} \mathrm{OS}\right) \mathrm{C}, \mathrm{H}, \mathrm{N}, \mathrm{S}$.

4-(4-Cyclohexylthiosemicarbazono)methyl-phenol (7). mp 223-225 ${ }^{\circ} \mathrm{C} ;{ }^{1} \mathrm{H}-\mathrm{NMR}$ (DMSO- $d_{6}$ ) $\delta$ 1.12-1.49 (m, 5 H), 1.53-1.65 (m, 1 H), 1.65-1.77 (m, 2 H), 1.81-1.93 (m, 2 H), 4.09-4.19 (m, 1 H), 6.79 $(\mathrm{d}, J=8.6 \mathrm{~Hz}, 2 \mathrm{H}), 7.59$ (d, $J=8.6 \mathrm{~Hz}, 2 \mathrm{H}), 7.83(\mathrm{~d}, J=8.6 \mathrm{~Hz}, 1 \mathrm{H}), 7.96$ (s, $1 \mathrm{H}), 9.86$ (s, $1 \mathrm{H}), 11.18$ $(\mathrm{s}, 1 \mathrm{H}) ;{ }^{13} \mathrm{C}-\mathrm{NMR}\left(\mathrm{DMSO}-d_{6}\right) \delta 24.87,25.11,31.87,52.40,115.54,125.01,129.00,142.58,159.23$, 175.36. Anal. $\left(\mathrm{C}_{14} \mathrm{H}_{19} \mathrm{~N}_{3} \mathrm{OS}\right) \mathrm{C}, \mathrm{H}, \mathrm{N}, \mathrm{S}$.

3-(4-Cyclohexylsemicarbazono)methyl-benzoic Acid (8). This compound was obtained in a similar manner as described except that cyclohexylsemicarbazide was used instead of cyclohexylthiosemicarbazide: $\operatorname{mp} 252-254{ }^{\circ} \mathrm{C} ;{ }^{1} \mathrm{H}-\mathrm{NMR}\left(\mathrm{DMSO}-d_{6}\right) \delta 1.02-1.41(\mathrm{~m}, 5 \mathrm{H}), 1.50-1.85$ (m, 5 H), 3.43-3.63 (m, 2 H), $6.58(\mathrm{~m}, 1 \mathrm{H}), 7.51(\mathrm{t}, J=7.7 \mathrm{~Hz}, 1 \mathrm{H}), 7.86-7.94(\mathrm{~m}, 2 \mathrm{H}), 7.99-8.06$ (s, 1 $\mathrm{H}), 8.07-8.13(\mathrm{~m}, 1 \mathrm{H}), 10.32(\mathrm{~s}, 1 \mathrm{H}) ;{ }^{13} \mathrm{C}-\mathrm{NMR}\left(\mathrm{DMSO}-d_{6}\right): \delta 24.93,25.20,32.91,48.14,127.49$, $128.89,129.67,130.28,131.31,135.09,138.72,154.53,167.01$.

2-[1-(4-Cyclohexylthiosemicarbazono)ethyl]-benzoic Acid (9). mp 197-199 ${ }^{\circ} \mathrm{C}$; ${ }^{1} \mathrm{H}-\mathrm{NMR}$ $\left(\mathrm{DMSO}-d_{6}\right) \delta 1.00-1.40(\mathrm{~m}, 5 \mathrm{H}), 1.45-1.89(\mathrm{~m}, 5 \mathrm{H}), 3.92-4.22(\mathrm{~m}, 1 \mathrm{H}), 2.50(\mathrm{~s}, 3 \mathrm{H}), 7.79-7.96$ (m, 3 $\mathrm{H}), 8.24(\mathrm{~d}, J=7.8 \mathrm{~Hz}, 1 \mathrm{H}), 9.15(\mathrm{br} \mathrm{s}, 1 \mathrm{H}) ;{ }^{13} \mathrm{C}-\mathrm{NMR}\left(\mathrm{DMSO}-d_{6}\right) \delta 18.93,25.02,25.50,32.04,53.08$, $126.05,126.23,127.83,130.25,131.94,139.92,143.75,159.94,181.15$.

2-(4-Cyclohexylthiosemicarbazono)methyl-benzoic Acid Methyl Ester (10). mp 160-162 ${ }^{\circ} \mathrm{C}$; ${ }^{1} \mathrm{H}-\mathrm{NMR}\left(\mathrm{DMSO}-d_{6}\right) \delta 1.13-1.53(\mathrm{~m}, 5 \mathrm{H}), 1.57-1.82(\mathrm{~m}, 3 \mathrm{H}), 2.07-2.15(\mathrm{~m}, 2 \mathrm{H}), 4.20-4.34(\mathrm{~m}, 1 \mathrm{H})$, $3.93(\mathrm{~s}, 3 \mathrm{H}), 7.32(\mathrm{~d}, J=8.0 \mathrm{~Hz}, 1 \mathrm{H}), 7.45(\mathrm{td}, J=7.7 / 1.4 \mathrm{~Hz}, 1 \mathrm{H}), 7.54(\mathrm{td}, J=7.7 / 1.3 \mathrm{~Hz}, 1 \mathrm{H}), 7.90$ $(\mathrm{dd}, J=6.8 /<1 \mathrm{~Hz}, 1 \mathrm{H}), 7.94(\mathrm{dd}, J=6.9 /<1 \mathrm{~Hz}, 1 \mathrm{H}), 8.68(\mathrm{~s}, 1 \mathrm{H}), 9.95(\mathrm{br}, 1 \mathrm{H}) ;{ }^{13} \mathrm{C}-\mathrm{NMR}$ (DMSO$\left.d_{6}\right) \delta 24.76,25.45,32.69,52.50,53.05,127.16,129.57,130.72,132.06,134.10,141.33,167.30,176.12$.

Sodium 2-(4-cyclohexylthiosemicarbazono)methyl-benzenesulfonate (11). mp $232-240 \quad{ }^{\circ} \mathrm{C}$; ${ }^{1} \mathrm{H}-\mathrm{NMR}\left(\mathrm{DMSO}-d_{6}\right) \delta 1.08-1.49(\mathrm{~m}, 5 \mathrm{H}), 1.53-1.64(\mathrm{~m}, 1 \mathrm{H}), 1.64-1.77$ (m, $\left.2 \mathrm{H}\right), 1.82-1.95(\mathrm{~m}, 2 \mathrm{H})$, 4.09-4.26 (m, $1 \mathrm{H}), 7.29-7.40$ (m, $2 \mathrm{H}), 7.77$ (m, $1 \mathrm{H}), 7.88(\mathrm{~d}, J=8.6 \mathrm{~Hz}), 8.03$ (m, $1 \mathrm{H}), 8.98$ (s, $1 \mathrm{H})$, $11.46(\mathrm{~s}, 1 \mathrm{H}) ;{ }^{13} \mathrm{C}-\mathrm{NMR}\left(\mathrm{DMSO}-d_{6}\right) \delta 24.82,25.08,31.80,52.35,126.17,126.63,128.48,128.61$, 130.07, 142.90, 146.82, 175.92. ES-MS: $m / z, 364\left([\mathrm{M}+\mathrm{H}]^{+}\right), 386\left([\mathrm{M}+\mathrm{Na}]^{+}\right)$. Anal. $\left(\mathrm{C}_{14} \mathrm{H}_{19} \mathrm{~N}_{3} \mathrm{OS}\right) \mathrm{C}, \mathrm{H}$, $\mathrm{N}, \mathrm{S}$

2-(4-Cyclohexylthiosemicarbazono)methyl-benzeneboronic Acid (12). mp 95-96 ${ }^{\circ} \mathrm{C}$; ${ }^{1} \mathrm{H}-\mathrm{NMR}$ 
$\left(\mathrm{DMSO}-d_{6}\right) \delta 1.07-1.81(\mathrm{~m}, 8 \mathrm{H}), 1.84-2.00(\mathrm{~m}, 2 \mathrm{H}), 4.15-4.34(\mathrm{~m}, 1 \mathrm{H}), 7.71(\mathrm{~d}, J=7.2 \mathrm{~Hz}, 1 \mathrm{H})$, 7.76-7.91 (m, $2 \mathrm{H}), 8.11(\mathrm{~d}, J=7.4 \mathrm{~Hz}, 1 \mathrm{H}), 8.26(\mathrm{~s}, 1 \mathrm{H}), 9.93(\mathrm{~d}, J=8.1 \mathrm{~Hz}, 1 \mathrm{H}), 10.57(\mathrm{~s}, 1$ $\mathrm{H}) ;{ }^{13} \mathrm{C}-\mathrm{NMR}\left(\mathrm{DMSO}-d_{6}\right) \delta 24.57,24.90,30.60,52.92,128.03,130.83,131.76,132.62,133.33,141.01$, 181.04 .

3-(1H-Tetrazol-5-yl)-benzaldehyde Cyclohexylthiosemicarbazone (13). mp 228-229 ${ }^{\circ} \mathrm{C}$; ${ }^{1} \mathrm{H}-\mathrm{NMR}$ $\left(\mathrm{DMSO}-d_{6}\right) \delta 1.07-1.50(\mathrm{~m}, 5 \mathrm{H}), 1.60(\mathrm{~m}, 1 \mathrm{H}), 1.66-1.79(\mathrm{~m}, 2 \mathrm{H}), 1.82-1.95(\mathrm{~m}, 2 \mathrm{H}), 4.19(\mathrm{~m}, 1 \mathrm{H})$, $7.65(\mathrm{t}, J=7.8 \mathrm{~Hz}, 1 \mathrm{H}), 7.99-8.07(\mathrm{~m}, 2 \mathrm{H}), 8.11(\mathrm{~d}, J=7.7 \mathrm{~Hz}, 1 \mathrm{H}), 8.15$ (s, $1 \mathrm{H}, \mathrm{CH}=\mathrm{N}), 8.25$ (br s, 1 $\mathrm{H}, \mathrm{H}-2), 11.53(\mathrm{~s}, 1 \mathrm{H},=\mathrm{N}-\mathrm{NH}) ;{ }^{13} \mathrm{C}-\mathrm{NMR}\left(\mathrm{DMSO}-d_{6}\right) \delta 24.90,25.10,31.77,52.74,124.78,135.23$ $\left(\mathrm{C}_{\mathrm{q}}\right), 126.11,128.03,129.09,129.76(\mathrm{C}-2,4,5,6), 141.04(\mathrm{CH}=\mathrm{N}), 175.84(\mathrm{C}=\mathrm{S})$. Anal. $\left(\mathrm{C}_{15} \mathrm{H}_{19} \mathrm{~N}_{7} \mathrm{~S}\right) \mathrm{C}, \mathrm{H}$, N, S.

2-(4-Butylthiosemicarbazono)methyl-benzoic Acid (14). This compound was obtained in a similar manner as described except that $n$-butylthiosemicarbazide was used instead of cyclohexylthiosemicarbazide: $\operatorname{mp} 218-220{ }^{\circ} \mathrm{C} ;{ }^{1} \mathrm{H}-\mathrm{NMR}\left(\mathrm{DMSO}-d_{6}\right) \delta 0.65-0.78(\mathrm{~m}, 4 \mathrm{H}), 2.45-2.54$ (m, $1 \mathrm{H}), 7.47(\mathrm{~m}, 1 \mathrm{H}), 7.56(\mathrm{~m}, 1 \mathrm{H}), 7.82(\mathrm{~m}, 1 \mathrm{H}), 8.15-8.27(\mathrm{~m}, 2 \mathrm{H}), 8.75(\mathrm{~s}, 1 \mathrm{H}), 11.62(\mathrm{~s}, 1 \mathrm{H})$, $13.23(\mathrm{br}, 1 \mathrm{H}) ;{ }^{13} \mathrm{C}-\mathrm{NMR}\left(\mathrm{DMSO}-d_{6}\right) \delta 6.50,26.99,127.23,129.21,130.78,134.09,130.78,134.09$, $142.27,168.15,178.63$.

2-(4-Hexylthiosemicarbazono)methyl-benzoic Acid (15). This compound was obtained in a similar manner as described except that $n$-hexylthiosemicarbazide was used instead of cyclohexylthiosemicarbazide: $\operatorname{mp} 218-220{ }^{\circ} \mathrm{C} ;{ }^{1} \mathrm{H}-\mathrm{NMR}\left(\mathrm{DMSO}-d_{6}\right) \delta 0.65-0.78(\mathrm{~m}, 4 \mathrm{H}), 2.45-2.54$ (m, $1 \mathrm{H}), 7.47$ (m, $1 \mathrm{H}), 7.56(\mathrm{~m}, 1 \mathrm{H}), 7.82$ (m, $1 \mathrm{H}), 8.15-8.27$ (m, $2 \mathrm{H}), 8.75(\mathrm{~s}, 1 \mathrm{H}), 11.62$ (s, $1 \mathrm{H})$, $13.23(\mathrm{br}, 1 \mathrm{H}) ;{ }^{13} \mathrm{C}-\mathrm{NMR}\left(\mathrm{DMSO}-d_{6}\right) \delta 6.50,26.99,127.23,129.21,130.78,134.09,130.78,134.09$, $142.27,168.15,178.63$.

2-(4-Cyclopropylthiosemicarbazono)methyl-benzoic Acid (16). This compound was obtained in a similar manner as described except that cyclopropylthiosemicarbazide was used instead of cyclohexylthiosemicarbazide: $\operatorname{mp} 218-220{ }^{\circ} \mathrm{C} ;{ }^{1} \mathrm{H}-\mathrm{NMR}\left(\mathrm{DMSO}-d_{6}\right) \delta 0.65-0.78(\mathrm{~m}, 4 \mathrm{H}), 2.45-2.54$ (m, $1 \mathrm{H}), 7.47$ (m, $1 \mathrm{H}), 7.56(\mathrm{~m}, 1 \mathrm{H}), 7.82$ (m, $1 \mathrm{H}), 8.15-8.27$ (m, $2 \mathrm{H}), 8.75(\mathrm{~s}, 1 \mathrm{H}), 11.62(\mathrm{~s}, 1 \mathrm{H})$, $13.23(\mathrm{br}, 1 \mathrm{H}) ;{ }^{13} \mathrm{C}-\mathrm{NMR}\left(\mathrm{DMSO}-d_{6}\right) \delta 6.50,26.99,127.23,129.21,130.78,134.09,130.78,134.09$, $142.27,168.15,178.63$.

2-(4-Cyclopentylthiosemicarbazono)methyl-benzoic Acid (17). This compound was obtained in a 
similar manner as described except that cyclopentylthiosemicarbazide was used instead of cyclohexylthiosemicarbazide: $\operatorname{mp} 220-222{ }^{\circ} \mathrm{C} ;{ }^{1} \mathrm{H}-\mathrm{NMR}\left(\mathrm{DMSO}-d_{6}\right) \delta 1.46-1.78(\mathrm{~m}, 6 \mathrm{H}), 1.85-2.03$ (m, 2 H), 4.54-4.71 (m, $1 \mathrm{H}), 7.48(\mathrm{~m}, 1 \mathrm{H}), 7.59(\mathrm{~m}, 1 \mathrm{H}), 7.82(\mathrm{~m}, 1 \mathrm{H}), 8.15(\mathrm{~m}, 1 \mathrm{H}), 8.01(\mathrm{~d}, J=8.1$ $\mathrm{Hz}, 1 \mathrm{H}), 8.75$ (s, $1 \mathrm{H}), 11.58$ (s, $1 \mathrm{H}), 13.20$ (br, $1 \mathrm{H}) ;{ }^{13} \mathrm{C}-\mathrm{NMR}$ (DMSO- $d_{6}$ ) $\delta$ 23.41, 31.66, 55.26, $127.21,129.24,129.85,131.54,130.85,134.02,142.29,168.20,176.58$.

2-(4-Cycloheptylthiosemicarbazono)methyl-benzoic Acid (18). This compound was obtained in a similar manner as described except that cycloheptylthiosemicarbazide was used instead of cyclohexylthiosemicarbazide: $\mathrm{mp} 235-237{ }^{\circ} \mathrm{C} ;{ }^{1} \mathrm{H}-\mathrm{NMR}\left(\mathrm{DMSO}-d_{6}\right) \delta 1.35-1.74(\mathrm{~m}, 10 \mathrm{H}), 1.82-1.98$ (m, 2 H), 4.26-4.43 (m, 1 H), $7.48(\mathrm{~m}, 1 \mathrm{H}), 7.59(\mathrm{~m}, 1 \mathrm{H}), 7.82(\mathrm{~m}, 1 \mathrm{H}), 8.13(\mathrm{~m}, 1 \mathrm{H}), 7.97(\mathrm{~d}, \mathrm{~J}=8.5$ $\mathrm{Hz}, 1 \mathrm{H}), 8.75$ (s, $1 \mathrm{H}), 11.54$ (s, $1 \mathrm{H}), 13.22$ (br, $1 \mathrm{H}) ;$ 13C-NMR (DMSO-d6) $\delta$ 23.88, 27.37, 33.85, $54.86,127.13,129.25,129.90,131.58,130.82,134.07,141.21,168.16,175.71$.

2-(4-Cyclooctylthiosemicarbazono)methyl-benzoic Acid (19). This compound was obtained in a similar manner as described except that cyclooctylthiosemicarbazide was used instead of cyclohexylthiosemicarbazide: mp $225-227{ }^{\circ} \mathrm{C} ;{ }^{1} \mathrm{H}-\mathrm{NMR}\left(\mathrm{DMSO}-d_{6}\right) \delta 1.44-1.81(\mathrm{~m}, 14 \mathrm{H}), 4.40-4.49$ (m, $1 \mathrm{H}), 7.48(\mathrm{~m}, 1 \mathrm{H}), 7.59(\mathrm{~m}, 1 \mathrm{H}), 7.82(\mathrm{~m}, 1 \mathrm{H}), 8.14(\mathrm{~m}, 1 \mathrm{H}), 8.02(\mathrm{~d}, J=8.5 \mathrm{~Hz}, 1 \mathrm{H}), 8.74(\mathrm{~s}, 1$ $\mathrm{H}), 11.56(\mathrm{~s}, 1 \mathrm{H}), 13.27$ (br, $1 \mathrm{H},) ;{ }^{13} \mathrm{C}-\mathrm{NMR}\left(\mathrm{DMSO}-d_{6}\right) \delta 25.68,25.22,26.53,31.96,53.51,127.20$, $129.32,129.97,131.68,130.79,134.14,141.20,168.21,175.62$.

2-(4-Phenylthiosemicarbazono)methyl-benzoic Acid (20). This compound was obtained in a similar manner as described except that phenylthiosemicarbazide was used instead of cyclohexylthiosemicarbazide: mp 222-224 ${ }^{\circ} \mathrm{C} ;{ }^{1} \mathrm{H}-\mathrm{NMR}$ (DMSO- $\left.d_{6}\right) \delta 0.82(\mathrm{~m}, 3 \mathrm{H}), 1.15-1.35$ (m, 28 H), $1.56(\mathrm{~m}, 2 \mathrm{H}), 3.49-3.57(\mathrm{~m}, 4 \mathrm{H}), 7.42-7.51(\mathrm{~m}, 1 \mathrm{H}), 7.52-7.61(\mathrm{~m}, 1 \mathrm{H}), 7.78-7.85(\mathrm{~m}, 1 \mathrm{H})$, 8.16-8.23 (m, $1 \mathrm{H}), 8.38-8.49(\mathrm{~m}, 1 \mathrm{H}), 8.76(\mathrm{~s}, 1 \mathrm{H}), 11.51(\mathrm{~s}, 1 \mathrm{H}) ;{ }^{13} \mathrm{C}-\mathrm{NMR}\left(\mathrm{DMSO}-d_{6}\right) \delta 13.99$, $22.13,26.36,28.72,28.82,29.03,31.32,43.56,127.04,129.33,130.08,131.60,131.04,134.31,141.14$, 168.29, 177.10 .

3-(4-Butylthiosemicarbazono)methyl-benzoic Acid (21). This compound was obtained in a similar manner as described except that $n$-butylthiosemicarbazide was used instead of cyclohexylthiosemicarbazide: mp $227-229{ }^{\circ} \mathrm{C} ;{ }^{1} \mathrm{H}-\mathrm{NMR}\left(\mathrm{DMSO}-d_{6}\right): \delta 0.99\left(\mathrm{~m}, 3 \mathrm{H}, \mathrm{CH}_{3}\right), 1.23-1.38$ (m, 2 H), 1.49-1.64 (m, 2 H), 3.49-3.64 (m, 2 H), 7.49-7.58 (m, 1 H), 7.91-7.95 (m, 1 H), 8.07-8.10 (m, $1 \mathrm{H}), 8.11(\mathrm{~s}, 1 \mathrm{H}), 8.50-8.60(\mathrm{~m}, 1 \mathrm{H}), 11.42(\mathrm{~s}, 1 \mathrm{H}) ;{ }^{13} \mathrm{C}-\mathrm{NMR}\left(\mathrm{DMSO}-d_{6}\right) \delta$ 13.77, 19.57, 31.01, 
$43.26,128.17,128.96,130.31,130.87,131.42,134.68,141.18,166.96,177.01$.

3-(4-Hexylthiosemicarbazono)methyl-benzoic Acid (22). This compound was obtained in a similar manner as described except that $n$-hexylthiosemicarbazide was used instead of cyclohexylthiosemicarbazide: mp $207-209{ }^{\circ} \mathrm{C} ;{ }^{1} \mathrm{H}-\mathrm{NMR}\left(\mathrm{DMSO}-d_{6}\right): \delta 0.79-0.92(\mathrm{~m}, 3 \mathrm{H}), 1.20-1.37$ (m, $6 \mathrm{H}), 1.48-1.65(\mathrm{~m}, 2 \mathrm{H}), 3.47-3.65(\mathrm{~m}, 2 \mathrm{H}), 7.53(\mathrm{~m}, J=8.7 \mathrm{~Hz}, 1 \mathrm{H}), 7.94$ (m, $1 \mathrm{H}), 8.06-8.14$ (m, $2 \mathrm{H}), 8.18(\mathrm{~s}, 1 \mathrm{H}), 8.54(\mathrm{~m}, 1 \mathrm{H}), 11.41(\mathrm{~s}, 1 \mathrm{H}) ;{ }^{13} \mathrm{C}-\mathrm{NMR}\left(\mathrm{DMSO}-d_{6}\right) \delta 13.86,22.01,26.00,28.76$, $31.02,43.55,128.15,128.92,130.30,130.85,131.44,134.67,141.16,166.96,176.98$.

3-(4-Cyclopentylthiosemicarbazono)methyl-benzoic Acid (23). This compound was obtained in a similar manner as described except that cyclopentylthiosemicarbazide was used instead of cyclohexylthiosemicarbazide: $\mathrm{mp} 238-240{ }^{\circ} \mathrm{C} ;{ }^{1} \mathrm{H}-\mathrm{NMR}\left(\mathrm{DMSO}-d_{6}\right): \delta 1.44-1.78(\mathrm{~m}, 6 \mathrm{H}), 1.85-2.02$ (m, $2 \mathrm{H}), 4.55-4.71(\mathrm{~m}, 1 \mathrm{H}), 7.50-7.58(\mathrm{~m}, 1 \mathrm{H}), 7.91-7.97$ (m, $1 \mathrm{H}), 8.08-8.18(\mathrm{~m}, 4 \mathrm{H}), 11.45$ (s, $1 \mathrm{H})$; ${ }^{13} \mathrm{C}-\mathrm{NMR}\left(\mathrm{DMSO}-d_{6}\right) \delta 23.47,31.67,55.45,128.46,129.01,130.41,130.75,131.40,134.56,141.51$, $169.94,176.58$.

3-(4-Cycloheptylthiosemicarbazono)methyl-benzoic Acid (24). This compound was obtained in a similar manner as described except that cycloheptylthiosemicarbazide was used instead of cyclohexylthiosemicarbazide: $\mathrm{mp} 236-238{ }^{\circ} \mathrm{C} ;{ }^{1} \mathrm{H}-\mathrm{NMR}\left(\mathrm{DMSO}-d_{6}\right): \delta 1.32-1.76(\mathrm{~m}, 10 \mathrm{H}), 1.79-1.97$ (m, $2 \mathrm{H}), 4.15-4.49$ (m, $2 \mathrm{H}), 7.54(\mathrm{~m}, 1 \mathrm{H}), 7.90-7.97$ (m, $1 \mathrm{H}), 8.01-8.16(\mathrm{~m}, 3 \mathrm{H}), 11.41$ (s, $1 \mathrm{H})$; ${ }^{13} \mathrm{C}-\mathrm{NMR}\left(\mathrm{DMSO}-d_{6}\right) \delta 23.96,27.36,33.85,55.02,128.34,129.00,130.37,130.71,131.40,134.54$, 141.36, 166.90, 175.67 .

2-(4-Cyclohexylthiosemicarbazono)methyl-6-hydroxy-benzoic Acid (25). mp 197-199 ${ }^{\circ}$; ${ }^{1} \mathrm{H}-\mathrm{NMR}\left(\mathrm{DMSO}-d_{6}\right) \delta 1.05-1.47(\mathrm{~m}, 5 \mathrm{H}), 1.53-1.78(\mathrm{~m}, 3 \mathrm{H}), 1.82-1.96(\mathrm{~m}, 2 \mathrm{H}), 4.10(\mathrm{~m}, 1 \mathrm{H}), 6.91$ (m, $1 \mathrm{H}, \mathrm{H}-5), 7.24-7.31(\mathrm{~m}, 2 \mathrm{H}, \mathrm{H}-3,4), 7.74$ (d, J = 8.4 Hz, $1 \mathrm{H}, \mathrm{NH}-\mathrm{cHex}), 8.12$ (s, $1 \mathrm{H}, \mathrm{CH}=\mathrm{N})$, $11.51(\mathrm{~s}, 1 \mathrm{H},=\mathrm{N}-\mathrm{NH}) ;{ }^{13} \mathrm{C}-\mathrm{NMR}\left(\mathrm{DMSO}-d_{6}\right) \delta 24.66,25.09,31.79,52.32,117.22,118.71(\mathrm{C}-3,5)$, $120.39(\mathrm{C}-1), 131.00(\mathrm{C}-4), 132.24(\mathrm{C}-2), 140.18(\mathrm{CH}=\mathrm{N}), 155.26(\mathrm{C}-6), 169.21(\mathrm{COOH}), 175.88$ $(\mathrm{C}=\mathrm{S})$. Anal. $\left(\mathrm{C}_{15} \mathrm{H}_{19} \mathrm{~N}_{3} \mathrm{O}_{3} \mathrm{~S}\right) \mathrm{C}, \mathrm{H}, \mathrm{N}, \mathrm{S}$.

2-(4-Cyclohexylthiosemicarbazono)methyl-6-methoxy-benzoic Acid (26). mp 226-228 ${ }^{\circ} \mathrm{C}$; ${ }^{1} \mathrm{H}-\mathrm{NMR}\left(\mathrm{DMSO}-d_{6}\right) \delta 1.02-1.49(\mathrm{~m}, 5 \mathrm{H}), 1.52-1.99(\mathrm{~m}, 5 \mathrm{H}), 4.11(\mathrm{~m}, 1 \mathrm{H}), 3.79\left(\mathrm{~s}, 3 \mathrm{H}, \mathrm{OCH}_{3}\right), 7.13$ (dd, $J=6.5 / 2.8 \mathrm{~Hz}, 1 \mathrm{H}, \mathrm{H}-5), 7.35-7.48$ (m, $2 \mathrm{H}, \mathrm{H}-3,4), 7.76$ (d, J= 8.4 Hz, $1 \mathrm{H}, \mathrm{HN}-\mathrm{cHex}), 8.02$ (s, 1 $\mathrm{H}, \mathrm{CH}=\mathrm{N}), 11.56(\mathrm{~s}, 1 \mathrm{H},=\mathrm{N}-\mathrm{NH}), 13.06(\mathrm{br}, 1 \mathrm{H}) ;{ }^{13} \mathrm{C}-\mathrm{NMR}\left(\mathrm{DMSO}-d_{6}\right) \delta 24.63,25.09,31.76,52.34$, 
55.94, 112.76, $119.86(\mathrm{C}-3,5), 123.65(\mathrm{C}-1), 130.00(\mathrm{C}-4), 131.20(\mathrm{C}-2), 138.95(\mathrm{CH}=\mathrm{N}), 155.63(\mathrm{C}-6)$, $168.15(\mathrm{COOH}), 175.92(\mathrm{C}=\mathrm{S})$.

6-(4-Cyclohexylthiosemicarbazono)methyl-2-hydroxy-3-methyl-benzoic Acid (27). mp 197-198 ${ }^{\circ} \mathrm{C} ;{ }^{1} \mathrm{H}-\mathrm{NMR}\left(\mathrm{DMSO}-d_{6}\right) \delta 1.13(\mathrm{~m}, 1 \mathrm{H}), 1.27(\mathrm{~m}, 2 \mathrm{H}), 1.40(\mathrm{~m}, 2 \mathrm{H}), 1.59(\mathrm{~m}, 1 \mathrm{H}), 1.72(\mathrm{~m}, 2 \mathrm{H})$, 1.87 (m, $2 \mathrm{H}), 2.19$ (s, $\left.3 \mathrm{H}, 3-\mathrm{CH}_{3}\right), 4.15(\mathrm{~m}, 1 \mathrm{H}), 7.29$ (d, J = 7.8 Hz, $\left.1 \mathrm{H}, \mathrm{H}-4\right), 7.35$ (d, J = 7.8 Hz, $1 \mathrm{H}$, $\mathrm{H}-5), 7.80$ (d, J = 8.5 Hz, $1 \mathrm{H}, \mathrm{NH}-\mathrm{cHex}), 8.45$ (s, $1 \mathrm{H}, \mathrm{CH}=\mathrm{N}), 11.46(\mathrm{~s}, 1 \mathrm{H},=\mathrm{N}-\mathrm{NH}) ;{ }^{13} \mathrm{C}-\mathrm{NMR}$ $\left(\mathrm{DMSO}-d_{6}\right) \delta 16.07\left(3-\mathrm{CH}_{3}\right), 24.76,25.08,31.79,52.39,115.95(\mathrm{C}-1), 118.83(\mathrm{C}-5), 127.44(\mathrm{C}-3)$, $131.86(\mathrm{C}-6), 133.17(\mathrm{C}-4), 141.95(\mathrm{CH}=\mathrm{N}), 156.61(\mathrm{C}-2), 171.12(\mathrm{COOH}), 175.77(\mathrm{C}=\mathrm{S})$. Anal. $\left(\mathrm{C}_{16} \mathrm{H}_{21} \mathrm{~N}_{3} \mathrm{O}_{3} \mathrm{~S}\right) \mathrm{C}, \mathrm{H}, \mathrm{N}, \mathrm{S}$.

6-(4-Cyclohexylthiosemicarbazono)methyl-2-methoxy-3-methyl-benzoic Acid (28). mp 206-208 ${ }^{\circ} \mathrm{C} ;{ }^{1} \mathrm{H}-\mathrm{NMR}\left(\mathrm{DMSO}-d_{6}\right) \delta 1.08-1.41(\mathrm{~m}, 5 \mathrm{H}), 1.50-2.20(\mathrm{~m}, 5 \mathrm{H}), 3.97-4.15(\mathrm{~m}, 1 \mathrm{H}), 2.30(\mathrm{~s}, 3 \mathrm{H})$, $3.75(\mathrm{~s}, 3 \mathrm{H}), 7.04(\mathrm{~d}, J=8.5 \mathrm{~Hz}, 1 \mathrm{H}), 7.23(\mathrm{~d}, J=8.8 \mathrm{~Hz}, 1 \mathrm{H}), 7.52(\mathrm{~d}, J=8.4 \mathrm{~Hz}, 1 \mathrm{H}), 8.24(\mathrm{~s}, 1 \mathrm{H})$, $11.50(\mathrm{~s}, 1 \mathrm{H}), 12.75(\mathrm{br}, 1 \mathrm{H}) ;{ }^{13} \mathrm{C}-\mathrm{NMR}\left(\mathrm{DMSO}-d_{6}\right) \delta 18.42,24.35,25.08,31.83,51.96,55.94,112.89$, $123.09,128.96,131.67,137.85,153.94,168.94,175.89$.

(E,Z)-2-(4-Cyclohexylthiosemicarbazono)methyl-6-hydroxy-3-methyl-benzoic Acid (29). mp $227-230{ }^{\circ} \mathrm{C}$; NMR-signals for the major isomer: ${ }^{1} \mathrm{H}-\mathrm{NMR}$ (DMSO- $\left.d_{6}\right) \delta 1.08-1.42(\mathrm{~m}, 5 \mathrm{H}), 1.48-1.64$ (m, $1 \mathrm{H}), 1.54-1.81(\mathrm{~m}, 2 \mathrm{H}), 1.82-2.05(\mathrm{~m}, 2 \mathrm{H}), 3.95-4.17(\mathrm{~m}, 1 \mathrm{H}), 2.25(\mathrm{~s}, 3 \mathrm{H}), 6.81(\mathrm{~d}, J=8.3 \mathrm{~Hz}, 1$ H), $7.07(\mathrm{~d}, J=8.3 \mathrm{~Hz}, 1 \mathrm{H}), 7.52(\mathrm{~d}, J=8.2 \mathrm{~Hz}, 1 \mathrm{H}), 8.23(\mathrm{~s}, 1 \mathrm{H}), 11.44(\mathrm{~s}, 1 \mathrm{H}) ;{ }^{13} \mathrm{C}-\mathrm{NMR}$ (DMSO$\left.d_{6}\right) \delta 18.43,24.38,25.08,31.84,51.95,116.97,121.15,127.27,129.25,131.74,138.60,152.40,169.55$, 175.83. Anal. $\left(\mathrm{C}_{16} \mathrm{H}_{21} \mathrm{~N}_{3} \mathrm{O}_{3} \mathrm{~S}\right) \mathrm{C}, \mathrm{H}, \mathrm{N}, \mathrm{S}$.

(E,Z)-2-(4-Cyclohexylthiosemicarbazono)methyl-6-methoxy-3-methyl-benzoic Acid (30). mp 214-217 ${ }^{\circ} \mathrm{C}$; NMR-signals for the major isomer: ${ }^{1} \mathrm{H}-\mathrm{NMR}\left(\mathrm{DMSO}-d_{6}\right) \delta 1.03-1.97(\mathrm{~m}, 10 \mathrm{H}), 4.05-4.25$ $(\mathrm{m}, 1 \mathrm{H}), 2.21(\mathrm{~s}, 3 \mathrm{H}), 3.82(\mathrm{~s}, 3 \mathrm{H}), 7.04(\mathrm{~d}, J=8.0 \mathrm{~Hz}, 1 \mathrm{H}), 7.23(\mathrm{~d}, J=7.9 \mathrm{~Hz}, 1 \mathrm{H}), 7.84(\mathrm{~d}, J=8.5$ $\mathrm{Hz}, 1 \mathrm{H}), 8.46$ (s, $1 \mathrm{H}), 11.59$ (s, $1 \mathrm{H}) ;{ }^{13} \mathrm{C}-\mathrm{NMR}\left(\mathrm{DMSO}-d_{6}\right) \delta 15.95,24.84,25.11,31.88,52.26,60.95$, $120.03,128.15,142.82,153.22,169.92,175.58$.

6-(4-Cyclohexylthiosemicarbazono)methyl-2-hydroxy-3-methoxy-benzoic Acid (31). mp 192-193 ${ }^{\circ} \mathrm{C} ;{ }^{1} \mathrm{H}-\mathrm{NMR}\left(\mathrm{DMSO}-d_{6}\right) \delta 1.09-1.44(\mathrm{~m}, 5 \mathrm{H}), 1.58(\mathrm{~m}, 1 \mathrm{H}), 1.65-1.78$ (m, $\left.2 \mathrm{H}\right), 1.83-1.96$ (m, $\left.2 \mathrm{H}\right)$, $3.84\left(\mathrm{~s}, 3 \mathrm{H}, \mathrm{OCH}_{3}\right), 4.10(\mathrm{~m}, 1 \mathrm{H}), 7.03,7.20(2 \mathrm{~d}, J=8.5 \mathrm{~Hz}, 2 \mathrm{H}, \mathrm{H}-4,5), 7.63(\mathrm{~d}, J=8.4 \mathrm{~Hz}, 1 \mathrm{H}$, $\mathrm{HN}-\mathrm{cHex}), 8.04(\mathrm{~s}, 1 \mathrm{H}, \mathrm{CH}=\mathrm{N}), 11.39(\mathrm{~s}, 1 \mathrm{H},=\mathrm{N}-\mathrm{NH}) ;{ }^{13} \mathrm{C}-\mathrm{NMR}\left(\mathrm{DMSO}-d_{6}\right) \delta 24.60,25.09,31.87$, 
52.13, $56.02\left(\mathrm{OCH}_{3}\right), 112.08,120.11(\mathrm{C}-4,5), 120.80,123.68(\mathrm{C}-1,6), 140.42(\mathrm{CH}=\mathrm{N}), 144.45,149.27$ $(\mathrm{C}-2,3), 168.89(\mathrm{COOH}), 175.65(\mathrm{C}=\mathrm{S})$.

6-(4-Cyclohexylthiosemicarbazono)methyl-2,3-dihydroxy-benzoic Acid (32). mp 206-208 ${ }^{\circ} \mathrm{C}$; ${ }^{1} \mathrm{H}-\mathrm{NMR}\left(\mathrm{DMSO}-d_{6}\right) \delta 1.01-2.06(\mathrm{~m}, 10 \mathrm{H}), 4.10(\mathrm{br}, 1 \mathrm{H}), 6.85,7.11(2 \mathrm{~d}, J=8.1 \mathrm{~Hz}, 2 \mathrm{H}, \mathrm{H}-4,5), 7.62$ (d, $J=8.2 \mathrm{~Hz}, 1 \mathrm{H}, \mathrm{HN}-\mathrm{cHex}), 8.05(\mathrm{~s}, 1 \mathrm{H}, \mathrm{CH}=\mathrm{N}), 10.00$ (br, $1 \mathrm{H}), 11.35$ (br s, $1 \mathrm{H},=\mathrm{N}-\mathrm{NH})$; ${ }^{13} \mathrm{C}-\mathrm{NMR}\left(\mathrm{DMSO}-d_{6}\right): \delta 24.64,25.09,31.89,52.12,115.83,120.05(\mathrm{C}-4,5), 120.82,122.54(\mathrm{C}-1,6)$, $140.92(\mathrm{CH}=\mathrm{N}), 143.93,147.46(\mathrm{C}-2,3), 169.25(\mathrm{COOH}), 175.54(\mathrm{C}=\mathrm{S})$. Anal. $\left(\mathrm{C}_{15} \mathrm{H}_{19} \mathrm{~N}_{3} \mathrm{O}_{4} \mathrm{~S}\right) \mathrm{C}, \mathrm{H}, \mathrm{N}$, S.

6-(4-Cyclohexylthiosemicarbazono)methyl-2,3-dimethoxy-benzoic Acid (33). mp 115-120 ${ }^{\circ} \mathrm{C}$ (foam); ${ }^{1} \mathrm{H}-\mathrm{NMR}\left(\mathrm{DMSO}-d_{6}\right) \delta 1.08-1.45(\mathrm{~m}, 5 \mathrm{H}), 1.59(\mathrm{~m}, 1 \mathrm{H}), 1.66-1.80(\mathrm{~m}, 2 \mathrm{H}), 1.83-1.98$ (m, 2 $\mathrm{H}), 4.09(\mathrm{~m}, 1 \mathrm{H}), 3.74,3.87\left(2 \mathrm{~s}, 6 \mathrm{H}, 2,3-\mathrm{OCH}_{3}\right), 7.12,7.48(2 \mathrm{~d}, J=8.7 \mathrm{~Hz}, 2 \mathrm{H}, \mathrm{H}-4,5), 7.61(\mathrm{~d}, J=$ $8.4 \mathrm{~Hz}, 1 \mathrm{H}, \mathrm{HN}-\mathrm{cHex}), 7.96(\mathrm{~s}, 1 \mathrm{H}, \mathrm{CH}=\mathrm{N}), 11.46(\mathrm{~s}, 1 \mathrm{H},=\mathrm{N}-\mathrm{NH}) ;{ }^{13} \mathrm{C}-\mathrm{NMR}$ (DMSO- $\left.d_{6}\right): \delta 24.60$, 25.08, 31.87, 52.17, 56.02, 60.93 (2 x OCH3), 113.11, 125.22 (C-4,5), 122.91, 128.89 (C-1,6), 139.12 $(\mathrm{CH}=\mathrm{N}), 144.96,153.55(\mathrm{C}-2,3), 167.92(\mathrm{COOH}), 175.71(\mathrm{C}=\mathrm{S})$.

3-(4-Cyclohexylthiosemicarbazono)methyl-2-hydroxy-benzoic Acid (34). mp 227-229 ${ }^{\circ} \mathrm{C}$; ${ }^{1} \mathrm{H}-\mathrm{NMR}\left(\mathrm{DMSO}-d_{6}\right) \delta 1.02-1.52(\mathrm{~m}, 5 \mathrm{H}), 1.60(\mathrm{~m}, 1 \mathrm{H}), 1.65-1.79(\mathrm{~m}, 2 \mathrm{H}), 1.81-1.97(\mathrm{~m}, 2 \mathrm{H}), 4.18$ (m, $1 \mathrm{H}), 6.97$ (t, J=7.8 Hz, 1 H, H-5), 7.84 (dd, J = 7.8/1.7 Hz, $1 \mathrm{H}, \mathrm{H}-6), 8.00$ (d, J = 8.4 Hz, $1 \mathrm{H}$, $\mathrm{NH}-\mathrm{cHex}), 8.30(\mathrm{dd}, J=7.7 / 1.7 \mathrm{~Hz}, 1 \mathrm{H}, \mathrm{H}-4), 8.42(\mathrm{~s}, 1 \mathrm{H}, \mathrm{CH}=\mathrm{N}), 11.47(\mathrm{~s}, 1 \mathrm{H},=\mathrm{N}-\mathrm{NH}) ;{ }^{13} \mathrm{C}-\mathrm{NMR}$ $\left(\mathrm{DMSO}-d_{6}\right) \delta 24.92,25.14,31.81,52.64,113.45,122.12(\mathrm{C}-1,3), 119.01(\mathrm{C}-5), 131.76,131.94(\mathrm{C}-4,6)$, $136.58(\mathrm{CH}=\mathrm{N}), 159.74(\mathrm{C}-2), 172.02,175.73(\mathrm{COOH}, \mathrm{C}=\mathrm{S})$. Anal. $\left(\mathrm{C}_{15} \mathrm{H}_{19} \mathrm{~N}_{3} \mathrm{O}_{3} \mathrm{~S}\right) \mathrm{C}, \mathrm{H}, \mathrm{N}, \mathrm{S}$.

5-(4-Cyclohexylthiosemicarbazono)methyl-2-hydroxy-benzoic Acid (35). mp 240-242 ${ }^{\circ} \mathrm{C}$; ${ }^{1} \mathrm{H}-\mathrm{NMR}\left(\mathrm{DMSO}-d_{6}\right) \delta 1.14(\mathrm{~m}, 1 \mathrm{H}), 1.20-1.33$ (m, $\left.2 \mathrm{H}\right), 1.34-1.48$ (m, $\left.2 \mathrm{H}\right), 1.59$ (m, $\left.1 \mathrm{H}\right), 1.65-1.76$ (m, $2 \mathrm{H}), 1.80-1.91(\mathrm{~m}, 2 \mathrm{H}), 4.17$ (m, $1 \mathrm{H}), 7.01$ (d, J = 8.7 Hz, $1 \mathrm{H}, \mathrm{H}-3), 7.93$ (d, J = 2.1 Hz, $1 \mathrm{H}, \mathrm{H}-6)$, $7.05(\mathrm{~d}, J=8.7 \mathrm{~Hz}, 1 \mathrm{H}, \mathrm{HN}-\mathrm{cHex}), 8.01(\mathrm{~s}, 1 \mathrm{H}, \mathrm{CH}=\mathrm{N}), 8.12(\mathrm{dd}, J=8.7 / 2.0 \mathrm{~Hz}, 1 \mathrm{H}, \mathrm{H}-4), 11.28(\mathrm{~s}, 1$ $\mathrm{H},=\mathrm{N}-\mathrm{NH}) ;{ }^{13} \mathrm{C}-\mathrm{NMR}\left(\mathrm{DMSO}-d_{6}\right) \delta 24.91,25.13,31.82,52.59,113.16(\mathrm{C}-1), 117.91(\mathrm{C}-3), 125.52$ (C-5), 130.30, $133.24(\mathrm{C}-4,6), 141.47(\mathrm{CH}=\mathrm{N}), 162.33(\mathrm{C}-2), 171.38,175.55$ (COOH, C=S). Anal. $\left(\mathrm{C}_{15} \mathrm{H}_{19} \mathrm{~N}_{3} \mathrm{O}_{3} \mathrm{~S}\right) \mathrm{C}, \mathrm{H}, \mathrm{N}, \mathrm{S}$.

3-(4-Cyclohexylthiosemicarbazono)methyl-4-hydroxy-benzoic Acid (36). mp >350 ${ }^{\circ} \mathrm{C}$; ${ }^{1} \mathrm{H}-\mathrm{NMR}$ $\left(\mathrm{DMSO}_{6}\right) \delta 1.08-1.49(\mathrm{~m}, 5 \mathrm{H}), 1.51-1.76(\mathrm{~m}, 3 \mathrm{H}), 1.82-1.95(\mathrm{~m}, 2 \mathrm{H}), 4.16(\mathrm{~m}, 1 \mathrm{H}), 6.94(\mathrm{~d}, J=8.6$ 
Hz, $1 \mathrm{H}, \mathrm{H}-5), 7.80$ (dd, $J=8.6 / 2.2 \mathrm{~Hz}, 1 \mathrm{H}, \mathrm{H}-6), 7.94$ (d, $J=8.4 \mathrm{~Hz}, 1 \mathrm{H}, \mathrm{HN}-\mathrm{cHex}), 8.32(\mathrm{~d}, J=2.2$ $\mathrm{Hz}, 1 \mathrm{H}, \mathrm{H}-2), 8.38$ (s, $1 \mathrm{H}, \mathrm{CH}=\mathrm{N}), 10.78$ (br s, $1 \mathrm{H}), 11.38$ (s, $1 \mathrm{H},=\mathrm{N}-\mathrm{NH}), 12.59$ (br, $1 \mathrm{H})$; ${ }^{13} \mathrm{C}-\mathrm{NMR}$ $\left(\mathrm{DMSO}-d_{6}\right) \delta 24.75,25.08,31.75,52.51,116.00(\mathrm{C}-5), 120.21,121.98(\mathrm{C}-1,3), 128.43,132.29(\mathrm{C}-2,6)$, $139.12(\mathrm{CH}=\mathrm{N}), 160.11(\mathrm{C}-4), 166.92(\mathrm{COOH}), 175.79(\mathrm{C}=\mathrm{S})$. Anal. $\left(\mathrm{C}_{15} \mathrm{H}_{19} \mathrm{~N}_{3} \mathrm{O}_{3} \mathrm{~S}\right) \mathrm{C}, \mathrm{H}, \mathrm{N}, \mathrm{S}$.

3-(4-Cyclohexylthiosemicarbazono)methyl-4-hydroxy-benzoic Acid Ethyl Ester (37). mp 209-211 ${ }^{\circ} \mathrm{C} ;{ }^{1} \mathrm{H}-\mathrm{NMR}\left(\mathrm{DMSO}-d_{6}\right) \delta 1.29(\mathrm{t}, J=7.1 \mathrm{~Hz}, 3 \mathrm{H}, \mathrm{CH} 3, \mathrm{OEt}), 1.08-1.74(\mathrm{~m}, 8 \mathrm{H}), 1.81-1.94$ $(\mathrm{m}, 2 \mathrm{H}), 4.16(\mathrm{~m}, 1 \mathrm{H}), 4.26(\mathrm{q}, J=7.1 \mathrm{~Hz}, 2 \mathrm{H}, \mathrm{CH} 2, \mathrm{OEt}), 6.97(\mathrm{~d}, J=8.6 \mathrm{~Hz}, 1 \mathrm{H}), 7.81(\mathrm{dd}, J=$ 8.6/2.2 Hz, $1 \mathrm{H}), 7.93$ (d, J=8.4 Hz, $1 \mathrm{H}), 8.34$ (d, $J=2.1 \mathrm{~Hz}, 1 \mathrm{H}), 8.37$ (s, $1 \mathrm{H}), 10.86$ (br. s, $1 \mathrm{H}), 11.43$ $(\mathrm{s}, 1 \mathrm{H}) ;{ }^{13} \mathrm{C}-\mathrm{NMR}\left(\mathrm{DMSO}-d_{6}\right) \delta 14.12,24.58,25.09,31.69,52.25,60.30,116.14(\mathrm{C}-5), 120.39,121.13$ $(\mathrm{C}-1,3), 128.18,131.93(\mathrm{C}-2,6), 138.64(\mathrm{CH}=\mathrm{N}), 160.31,165.29(\mathrm{C}-4, \mathrm{COOH}), 175.83(\mathrm{C}=\mathrm{S})$.

Sodium 3-(4-Cyclohexylthiosemicarbazono)methyl-4-hydroxy-benzenesulfonate (38). mp >340 ${ }^{\circ} \mathrm{C} ;{ }^{1} \mathrm{H}-\mathrm{NMR}\left(\mathrm{DMSO}-d_{6}\right) \delta 1.07-1.47(\mathrm{~m}, 5 \mathrm{H}), 1.50-1.77(\mathrm{~m}, 3 \mathrm{H}), 1.84-1.98(\mathrm{~m}, 2 \mathrm{H}), 4.15(\mathrm{~m}, 1 \mathrm{H})$, $6.81(\mathrm{~d}, J=8.4 \mathrm{~Hz}, 1 \mathrm{H}, \mathrm{H}-5), 7.47$ (dd, $J=8.4 / 2.1 \mathrm{~Hz}, 1 \mathrm{H}, \mathrm{H}-6), 7.79$ (d, $J=8.4 \mathrm{~Hz}, 1 \mathrm{H}, \mathrm{HN}-\mathrm{cHex})$, $7.91(\mathrm{~d}, J=2.1 \mathrm{~Hz}, 1 \mathrm{H}, \mathrm{H}-2), 8.37$ (s, $1 \mathrm{H}, \mathrm{CH}=\mathrm{N}), 10.13$ (br, $1 \mathrm{H}), 11.34$ (s, $1 \mathrm{H}) ;{ }^{13} \mathrm{C}-\mathrm{NMR}$ (DMSO$\left.d_{6}\right) \delta 24.74,25.00,31.84,52.41,115.12(\mathrm{C}-5), 118.83(\mathrm{C}-3), 124.14128 .74(\mathrm{C}-2,6), 139.82(\mathrm{C}-1)$, $140.39(\mathrm{CH}=\mathrm{N}), 156.53(\mathrm{C}-4), 175.66(\mathrm{C}=\mathrm{S})$. Anal. $\left(\mathrm{C}_{14} \mathrm{H}_{18} \mathrm{~N}_{3} \mathrm{NaO}_{4} \mathrm{~S}\right) \mathrm{C}, \mathrm{H}, \mathrm{N}, \mathrm{S}$. 
Elemental analysis data for selected compounds

\begin{tabular}{|c|c|c|c|c|c|c|}
\hline Compd & Formula & & C & H & $\mathbf{N}$ & S \\
\hline \multirow[t]{2}{*}{2} & $\mathrm{C}_{15} \mathrm{H}_{19} \mathrm{~N}_{3} \mathrm{O}_{2} \mathrm{~S}$ & calcd & 58.99 & 6.27 & 13.76 & 10.50 \\
\hline & & found & 58.63 & 6.31 & 13.35 & 10.10 \\
\hline \multirow[t]{2}{*}{3} & $\mathrm{C}_{15} \mathrm{H}_{19} \mathrm{~N}_{3} \mathrm{O}_{2} \mathrm{~S}$ & calcd & 58.99 & 6.27 & 13.76 & 10.50 \\
\hline & & found & 58.90 & 6.29 & 13.47 & 10.23 \\
\hline \multirow[t]{2}{*}{4} & $\mathrm{C}_{15} \mathrm{H}_{19} \mathrm{~N}_{3} \mathrm{O}_{2} \mathrm{~S}$ & calcd & 58.99 & 6.27 & 13.76 & 10.50 \\
\hline & & found & 58.72 & 6.32 & 13.48 & 10.15 \\
\hline \multirow[t]{2}{*}{5} & $\mathrm{C}_{14} \mathrm{H}_{19} \mathrm{~N}_{3} \mathrm{OS}$ & calcd & 60.18 & 7.58 & 15.04 & 11.48 \\
\hline & & found & 59.80 & 7.65 & 14.74 & 11.15 \\
\hline \multirow[t]{2}{*}{6} & $\mathrm{C}_{14} \mathrm{H}_{19} \mathrm{~N}_{3} \mathrm{OS}$ & calcd & 60.18 & 7.58 & 15.04 & 11.48 \\
\hline & & found & 59.86 & 7.68 & 14.81 & 11.23 \\
\hline \multirow[t]{2}{*}{7} & $\mathrm{C}_{14} \mathrm{H}_{19} \mathrm{~N}_{3} \mathrm{OS}$ & calcd & 60.18 & 7.58 & 15.04 & 11.48 \\
\hline & & found & 59.83 & 7.71 & 14.71 & 11.19 \\
\hline \multirow[t]{2}{*}{11} & $\mathrm{C}_{14} \mathrm{H}_{18} \mathrm{~N}_{3} \mathrm{NaO}_{3} \mathrm{~S}$ & calcd & 46.27 & 4.99 & 11.56 & 17.65 \\
\hline & & found & 46.19 & 5.36 & 11.47 & 17.24 \\
\hline \multirow[t]{2}{*}{13} & $\mathrm{C}_{15} \mathrm{H}_{19} \mathrm{~N}_{7} \mathrm{~S}$ & calcd & 54.69 & 5.81 & 29.76 & 9.73 \\
\hline & & found & 54.35 & 5.80 & 29.90 & 9.87 \\
\hline \multirow[t]{2}{*}{25} & $\mathrm{C}_{15} \mathrm{H}_{19} \mathrm{~N}_{3} \mathrm{O}_{3} \mathrm{~S}$ & calcd & 56.06 & 5.96 & 13.07 & 9.98 \\
\hline & & found & 55.72 & 6.14 & 12.82 & 9.61 \\
\hline \multirow[t]{2}{*}{27} & $\mathrm{C}_{16} \mathrm{H}_{21} \mathrm{~N}_{3} \mathrm{O}_{3} \mathrm{~S}$ & calcd & 57.29 & 6.31 & 12.53 & 9.56 \\
\hline & & found & 57.09 & 6.43 & 12.18 & 9.14 \\
\hline \multirow[t]{2}{*}{29} & $\mathrm{C}_{16} \mathrm{H}_{21} \mathrm{~N}_{3} \mathrm{O}_{3} \mathrm{~S}$ & calcd & 57.29 & 6.31 & 12.53 & 9.56 \\
\hline & & found & 57.23 & 6.42 & 12.53 & 9.23 \\
\hline \multirow[t]{2}{*}{32} & $\mathrm{C}_{15} \mathrm{H}_{19} \mathrm{~N}_{3} \mathrm{O}_{4} \mathrm{~S}$ & calcd & 53.40 & 5.68 & 12.45 & 9.50 \\
\hline & & found & 53.04 & 5.52 & 12.20 & 9.78 \\
\hline \multirow[t]{2}{*}{34} & $\mathrm{C}_{15} \mathrm{H}_{19} \mathrm{~N}_{3} \mathrm{O}_{3} \mathrm{~S}$ & calcd & 56.06 & 5.96 & 13.07 & 9.98 \\
\hline & & found & 55.79 & 6.06 & 12.76 & 10.06 \\
\hline \multirow[t]{2}{*}{35} & $\mathrm{C}_{15} \mathrm{H}_{19} \mathrm{~N}_{3} \mathrm{O}_{3} \mathrm{~S}$ & calcd & 56.06 & 5.96 & 13.07 & 9.98 \\
\hline & & found & 55.85 & 6.12 & 12.82 & 9.61 \\
\hline \multirow[t]{2}{*}{36} & $\mathrm{C}_{15} \mathrm{H}_{19} \mathrm{~N}_{3} \mathrm{O}_{3} \mathrm{~S}$ & calcd & 56.06 & 5.96 & 13.07 & 9.98 \\
\hline & & found & 55.90 & 6.35 & 12.84 & 9.56 \\
\hline \multirow[t]{2}{*}{38} & $\mathrm{C}_{14} \mathrm{H}_{18} \mathrm{~N}_{3} \mathrm{NaO}_{4} \mathrm{~S}$ & calcd & 44.32 & 4.78 & 11.07 & 16.90 \\
\hline & & found & 43.99 & 5.02 & 11.23 & 17.15 \\
\hline \multirow[t]{2}{*}{39} & $\mathrm{C}_{31} \mathrm{H}_{30} \mathrm{~N}_{4} \mathrm{O}_{2}$ & calcd & 75.89 & 6.16 & 11.42 & - \\
\hline & & found & 75.74 & 6.25 & 11.15 & - \\
\hline \multirow[t]{2}{*}{40} & $\mathrm{C}_{8} \mathrm{H}_{6} \mathrm{~N}_{4} \mathrm{O}$ & calcd & 55.17 & 3.47 & 32.17 & - \\
\hline & & found & 55.21 & 3.51 & 31.83 & - \\
\hline \multirow[t]{2}{*}{45} & $\mathrm{C}_{8} \mathrm{H}_{6} \mathrm{O}_{4}$ & calcd & 57.84 & 3.64 & - & \\
\hline & & found & 57.61 & 3.71 & - & \\
\hline \multirow[t]{2}{*}{46} & $\mathrm{C}_{9} \mathrm{H}_{8} \mathrm{O}_{4}$ & calcd & 60.00 & 4.48 & - & - \\
\hline & & found & 60.02 & 4.47 & - & - \\
\hline \multirow[t]{2}{*}{47} & $\mathrm{C}_{9} \mathrm{H}_{8} \mathrm{O}_{4}$ & calcd & 60.00 & 4.48 & - & - \\
\hline & & found & 59.91 & 4.52 & - & - \\
\hline \multirow[t]{2}{*}{48} & $\mathrm{C}_{9} \mathrm{H}_{8} \mathrm{O}_{5}$ & calcd & 55.11 & 4.11 & - & - \\
\hline & & found & 55.02 & 4.19 & - & - \\
\hline 49 & $\mathrm{C}_{8} \mathrm{H}_{6} \mathrm{O}_{5}$ & calcd & 52.76 & 3.32 & - & - \\
\hline & & found & 52.82 & 3.27 & - & - \\
\hline
\end{tabular}




\section{References}

1. Chenard, B. L.; Dolson, M. G.; Sercel, A. D.; Swenton, J. S. Annelation reactions of quinone monoketals. Studies directed at an efficient synthesis of anthracyclinones. J. Org. Chem. 1984, 49, 318325.

2. Hoffmann, B.; Lackner, H. Strukturmodifizierte Isokalafungine und Isonanaomycine. Liebigs Ann. 1995, 87-94.

3. Hullar, T. L.; Failla, D. L. Pyridoxyl phosphate. II. Benzene analogs. 2-Formylphenoxyacetic acids as potential antimetabolites of pyridoxyl phosphate. J. Med. Chem. 1969, 12, 420-424.

4. Sunamoto, J.; Iwamoto, K.; Akutagawa, M.; Nagase, M.; Kondo, H. Rate control by restricting mobility of substrate in specific reaction field. Negative photochromism of water-soluble spiropyran in AOT reversed micelles. J. Am. Chem. Soc. 1982, 104, 4904-4907.

5. Hagemann, H. Carbonic acid derivatives. In Houben-Weyl; 4th ed.; Georg Thieme: Stuttgart, 1983. 